\title{
Spectrochemical differentiation of meningioma tumours based on attenuated total reflection Fourier-transform infrared (ATR-FTIR) spectroscopy
}

\author{
Taha Lilo $^{1,2}$ - Camilo L. M. Morais ${ }^{2}$ - Katherine M. Ashton ${ }^{3} \cdot$ Ana Pardilho $^{1} \cdot$ Charles Davis $^{2} \cdot$ Timothy P. Dawson $^{3}$. \\ Nihal Gurusinghe ${ }^{1}$ - Francis L. Martin ${ }^{2}$
}

Received: 1 October 2019 /Revised: 11 November 2019 / Accepted: 5 December 2019 / Published online: 21 December 2019

(C) The Author(s) 2019

\begin{abstract}
Meningiomas are the commonest types of tumours in the central nervous system (CNS). It is a benign type of tumour divided into three WHO grades (I, II and III) associated with tumour growth rate and likelihood of recurrence, where surgical outcomes and patient treatments are dependent on the meningioma grade and histological subtype. The development of alternative approaches based on attenuated total reflection Fourier-transform infrared (ATR-FTIR) spectroscopy could aid meningioma grade determination and its biospectrochemical profiling in an automated fashion. Herein, ATR-FTIR in combination with chemometric techniques is employed to distinguish grade I, grade II and grade I meningiomas that re-occurred. Ninety-nine patients were investigated in this study where their formalin-fixed paraffin-embedded (FFPE) brain tissue samples were analysed by ATRFTIR spectroscopy. Subsequent classification was performed via principal component analysis plus linear discriminant analysis (PCA-LDA) and partial least squares plus discriminant analysis (PLS-DA). PLS-DA gave the best results where grade I and grade II meningiomas were discriminated with $79 \%$ accuracy, $80 \%$ sensitivity and $73 \%$ specificity, while grade I versus grade I recurrence and grade II versus grade I recurrence were discriminated with $94 \%$ accuracy (94\% sensitivity and specificity) and $97 \%$ accuracy ( $97 \%$ sensitivity and $100 \%$ specificity), respectively. Several wavenumbers were identified as possible biomarkers towards tumour differentiation. The majority of these were associated with lipids, protein, DNA/RNA and carbohydrate alterations. These findings demonstrate the potential of ATR-FTIR spectroscopy towards meningioma grade discrimination as a fast, low-cost, non-destructive and sensitive tool for clinical settings.
\end{abstract}

Keywords Meningioma $\cdot$ Infrared spectroscopy $\cdot$ ATR-FTIR $\cdot$ Chemometrics

\section{Introduction}

Meningioma and glioma tumours constitute the majority of primary brain cancers [1]. Gliomas are a more aggressive

Electronic supplementary material The online version of this article (https://doi.org/10.1007/s00216-019-02332-w) contains supplementary material, which is available to authorized users.

Francis L. Martin

flmartin@uclan.ac.uk

1 Department of Neurosurgery, Royal Preston Hospital, Lancashire Teaching Hospitals NHS Trust, Preston PR2 9HT, UK

2 School of Pharmacy and Biomedical Sciences, UCLan, Preston PR1 2HE, UK

3 Department of Neuropathology, Royal Preston Hospital, Lancashire Teaching Hospitals NHS Trust, Preston PR2 9HT, UK and intrinsic type of tumour, which comprise neuroepithelial tumours originating from the glial or supporting cells of the central nervous system (CNS) [2]. Meningiomas are the commonest type of brain tumours, showing differentiation towards the meninges surrounding the brain and the spinal cord [3]. They are slow-growing extrinsic tumours with variable prognosis, occasionally growing to a very large size. The majority occur in a supratentorial location, while a few can arise in the posterior cranial fossa and, rarely, as extracranial meningiomas [4]. They often manifest as single or sporadic lesions, producing non-descript symptoms. The symptoms are variable in nature depending on the location and the size of the lesion. The most common presenting symptom is headache. However, symptoms may include any sensory and/or motor deficits and gait disturbance.

Multiple meningiomas are commonly associated with neurofibromatosis type II [5]. Meningiomas can be 
divided into WHO grade I (benign), grade II (atypical) and grade III (anaplastic) [3]. Grade I meningiomas are the commonest type of tumours, with slower growth and lower likelihood of recurrence, exhibiting histological patterns other than papillary, chordoid, clear cell or rhabdoid [6]; grade II meningiomas also have a slower growth but higher likelihood of recurrence, exhibiting 3 of the following parameters (macronuclei, spontaneous necrosis, hypercellularity, small cell formation, sheeting architecture), and have clear cell or chordoid cell types [6]; and grade III meningiomas are a very rare type of tumour with fast growing rate and much higher likelihood of recurrence, being cancerous aggressive, and histologically, they can resemble other tumours (melanomas, carcinomas and sarcomas) [6]. Accurate histological assessment is important since surgical outcomes and treatment are dependent on the meningioma grade and subtypes [5]. Moreover, the degree of surgical resection and Simpson grading have an important role also in patient outcome.

The pathogenesis and molecular genetics of meningioma are not very well understood; however, signalling pathways and growth factor alterations have been reported [6]. For example, cell cycle dysregulation and telomerase activation have been recognised as important steps in meningioma progression, where telomerase dynamics, cell cycle control and the mechanisms responsible for deoxyribonucleic acid damage control are highly entwined [6, 7]. Some WHO grade I meningiomas are linked to mutations of the NF2 gene on chromosome 22 (location q12.2), which leads to the condition known as neurofibromatosis type II where benign tumour proliferates through the CNS [6]. NF2 codes for the protein merlin that acts as a tumour suppressor in many different cell types. This protein interacts with the intermolecular amino-terminal domain and the carboxyl-terminal domain through phosphorylation that also controls the binding to its effector proteins [6]. In WHO grade I meningiomas, other proteins of 4.1 family members can also be downregulated, such as 4.1B (DAL-1) [6, 8]. Additionally, epidermal growth factor receptors (EGFRs) tend be overexpressed in grade I meningiomas, and the platelet-derived growth factor receptor beta (PDGFRB) gene is upregulated and overexpressed in this type of tumour [6]. On the other hand, WHO grade II meningiomas are characterised by chromosome mutations (mainly deletions) $[6,9]$, such as mutations in the $1 p$ and $14 q$ regions responsible for housing tumour suppressor genes, and further alterations in chromosome 1 [6]. Alterations in chromosome 10, including the PTEN gene located near the $\mathrm{p} 23.3$ region of chromosome 10, and other genetic non-NF2 aberrations are associated with higher-grade meningiomas [6,9]. PTEN is responsible for tumour suppression and production of the phosphatidylinositol-3,4,5-triphosphate 3-phosphate protein negatively that regulates the AKT/PKG pathway that has been linked to the pathogenesis and proliferation of meningiomas and other tumours [6,9].

Infrared (IR) spectroscopy is a powerful technique to investigate biological materials [10]. The interaction of IR with the biochemical molecules that make up a tissue sample generates a spectrochemical fingerprint, allowing one to extract both quantitative and qualitative information. The IR signal, obtained by a change of the molecular dipole moment, reflects vibrational movements by the chemical bonds in the material, such as stretching and bending vibrations. This signal is specific for each type of sample and can be used to diagnose samples and to identify possible spectral biomarkers associated with tumour appearance and differentiation [11]. The signal within the fingerprint region $\left(1800-900 \mathrm{~cm}^{-1}\right)$ contains important spectrochemical signatures of key biomolecules, such as lipids $\left(\mathrm{C}=\mathrm{O}\right.$ symmetric stretching at $1750 \mathrm{~cm}^{-1}, \mathrm{CH}_{2}$ bending at $1470 \mathrm{~cm}^{-1}$ ), proteins (Amide I at $1650 \mathrm{~cm}^{-1}$, Amide II at $1550 \mathrm{~cm}^{-1}$, Amide III at $1260 \mathrm{~cm}^{-1}$ ), nucleic acids (asymmetric phosphate stretching at $1225 \mathrm{~cm}^{-1}$, symmetric phosphate stretching at $\left.1080 \mathrm{~cm}^{-1}\right)$, carbohydrates $(\mathrm{CO}-\mathrm{O}-\mathrm{C}$ symmetric stretching at $\left.1155 \mathrm{~cm}^{-1}\right)$, glycogen $(\mathrm{C}-\mathrm{O}$ stretching at $\left.1030 \mathrm{~cm}^{-1}\right)$ and protein phosphorylation $\left(970 \mathrm{~cm}^{-1}\right)[10,12$, $13]$.

In order to obtain meaningful and reliable information, the IR spectra within the fingerprint region are processed through specific computational techniques, known as chemometrics. The spectral data initially undergo preprocessing techniques to correct the baseline and to remove possible physical variations not related to disease changes, and then chemometric models are built and validated, whereby possible spectral biomarkers as well as sensitivity and specificity metrics can be obtained [14]. Multivariate classification models, such as principal component analysis plus linear discriminant analysis (PCALDA) [15] and partial least squares plus discriminant analysis (PLS-DA) [16], are commonly employed to process IR spectral data, since these techniques allow to extract relevant spectral features associated with tumour differentiation and also to classify the samples into groups in a predictive fashion.

Gliomas comprise neuroepithelial tumours differentiating towards the glial or supporting cells of the CNS [2]. They are broadly classified into glioblastomas, astrocytomas, oligodendrogliomas, ependyomas and glioneuronal tumours [2]. Gliomas have been widely investigated using IR spectroscopy [16-23], while meningiomas have attracted relatively little attention [1, 24]. Meningiomas represent $20 \%$ to $35 \%$ of all primary intracranial tumours [4], and determining their WHO grade is essential to define appropriate treatment pathways. Herein, IR spectroscopy was applied to distinguish WHO grade I, II and I meningioma tumours that recurred. 


\section{Materials and methods}

\section{Samples and spectral acquisition}

Ninety-nine $10-\mu \mathrm{m}$-thick formalin-fixed paraffin-embedded (FFPE) brain tissue samples $(70$ WHO grade I meningiomas, $24 \mathrm{WHO}$ grade II meningiomas and $5 \mathrm{WHO}$ grade I meningiomas that re-occurred) were analysed by a Bruker Tensor 27 FTIR spectrometer with Helios ATR attachment (Bruker Optics Ltd., Coventry, UK). All samples were sourced from the Brain Tumour North West (BTNW) biobank (National Research Ethics Service's ethics approval NRES14/ EE/1270). All experiments were approved by the STEMH (Science, Technology, Engineering, Medicine and Health) ethics committee at the University of Central Lancashire (STEMH 917). The H\&E images for all samples are available upon reasonable request to the BTWN biobank; the sample details are depicted in Electronic Supplementary Material (ESM) Table S1. The sampling area, defined by an internal reflective element (diamond crystal), was approximately $250 \mu \mathrm{m} \times 250 \mu \mathrm{m}$. Samples were placed onto aluminiumcovered slides [25], which, in turn, were affixed onto a moving platform with the sample facing up. De-parrafinisation was performed prior to commencing measurements using local protocols using xylene and ethanol [10]. Moving the platform upward allowed the specimen to contact the diamond crystal for spectral acquisition. Spectral resolution was $8 \mathrm{~cm}^{-1}$, over the range between 4000 and $400 \mathrm{~cm}^{-1}$, with 32 co-addition scans. Ten spectra were collected per tissue sample in different random locations to minimise bias. After each sample, the ATR crystal was cleaned with distilled water and a new background spectrum was acquired to take into account ambient changes before the next sampling. The time to analyse each tissue sample was approximately $10 \mathrm{~min}$.

\section{Data analysis}

The spectral data analysis was performed within a MATLAB R2014b environment (MathWorks, Natick, USA) using the Classification Toolbox for MATLAB [26]. The biofingerprint spectra (1800-900 $\mathrm{cm}^{-1}$ ) were pre-processed by SavitzkyGolay $2^{\text {nd }}$ derivative (window of 7 points, $2^{\text {nd }}$-order polynomial fit) and vector normalisation, a common pre-process employed in biological-derived spectral data for correcting random noise and baseline distortions and to improve the signal-to-noise ratio $[10,14]$. An outlier test was performed using Hotelling's $T^{2}$ versus $Q$ residual test [14], and no spectral outlier was observed in the dataset (see ESM Fig. S3). Thereafter, the samples for grade I and grade II meningiomas were divided into training (70\% of samples) and validation $(30 \%)$ sets using the Kennard-Stone uniform sample selection algorithm [27]. Cross-validated PCA-LDA and PLS-DA were built using venetian blinds cross-validation with 10 data splits.
PCA-LDA is a supervised discriminant analysis algorithm based on a principal component analysis (PCA) model followed by a linear discriminant analysis (LDA) classifier [15] Initially, the pre-processed spectral data is reduced by PCA to a small number of principal components (PCs) accounting for the majority of the data explained variance [28]. Each PC is composed of scores and loadings: the first representing the variance on sample direction, thus being used to assess similarities/dissimilarities between samples, and the latter representing the variance on wavenumber direction, being used to find important spectral biomarkers. Then, a LDA model is built using the PCA scores, where the samples are assigned to classes based on a Mahalanobis distance calculation [15]. PLS-DA is another very popular supervised discriminant analysis technique that combines feature extraction and classification in a single routine [16]. In PLS-DA, a partial least squares (PLS) model is applied to the data reducing the pre-processed spectral data to a few numbers of latent variables (LVs); however, different from PCA-LDA, the input class labels for the training samples (e.g. +1 or -1 ) are used during this process, since PLS maximises the co-variation between the spectral information and the sample category. The samples are assigned to classes based on a straight line that divides the classes' spaces [16].

\section{Model evaluation}

The classification models were validated through the calculation of the accuracy, sensitivity and specificity in the validation set. Accuracy represents the total number of samples correctly classified considering true and false negatives, sensitivity represents the proportion of positive samples that are correctly classified and specificity represents the proportion of negative samples that are correctly classified [29]. These metrics are calculated as follows:

Accuracy $(\%)=\frac{\mathrm{TP}+\mathrm{TN}}{\mathrm{TP}+\mathrm{FP}+\mathrm{TN}+\mathrm{FN}} \times 100$

Sensitivity $(\%)=\frac{\mathrm{TP}}{\mathrm{TP}+\mathrm{FN}} \times 100$

Specificity $(\%)=\frac{\mathrm{TN}}{\mathrm{TN}+\mathrm{FP}} \times 100$

where TP stands for true positives, TN for true negatives, FP for false positives and $\mathrm{FN}$ for false negatives.

\section{Results}

This study is composed of 99 patients separated into 3 groups: grade I meningiomas ( $n=70,700$ spectra), grade II meningiomas ( $n=24,240$ spectra) and grade I meningiomas that reoccurred ( $n=5,50$ spectra) (see ESM Table S1). Sample 
groups were pre-defined based on histopathologic evidence before spectral acquisition. Figure 1 shows an example of $\mathrm{H} \& \mathrm{E}$ slide for WHO grade I and grade II meningiomas. The raw and pre-processed (Savitzky-Golay $2^{\text {nd }}$ derivative and vector normalisation) IR spectra for each sample class are shown in Fig. 2 a-c and in Figs. S1 and S2 (see ESM). Grade I and grade II meningiomas exhibit higher levels of variability in comparison with grade I recurrence most likely due to the smaller number of grade I recurrence spectra (Fig. 2b). The difference-between-mean (DBM) spectrum for grade II (+ coefficients) and grade I (- coefficients) meningiomas is shown in Fig. 2 d, where 15 spectral markers were found with absolute coefficient intensity $>0.01$ : $1725 \mathrm{~cm}^{-1}$ (lower coefficient in grade II, $\mathrm{C}=\mathrm{O}$ stretching in fatty acids), $1708 \mathrm{~cm}^{-1}$ (lower coefficient in grade II, $\mathrm{C}=\mathrm{O}$ stretching in thymine), $1698 \mathrm{~cm}^{-1}$ (higher coefficient in grade II, $\mathrm{C}_{2}=\mathrm{O}$ stretching in guanine), $1663 \mathrm{~cm}^{-1}$ (lower coefficient in grade II, $\mathrm{C}=\mathrm{O}$ stretching in cytosine), $1639 \mathrm{~cm}^{-1}$ (lower coefficient in grade II, Amide I), $1624 \mathrm{~cm}^{-1}$ (higher coefficient in grade II, base carbonyl stretching and ring breathing mode in nucleic acids), $1604 \mathrm{~cm}^{-1}$ (lower coefficient in grade II, adenine vibration in DNA), $1562 \mathrm{~cm}^{-1}$ (higher coefficient in grade II, ring base), $1550 \mathrm{~cm}^{-1}$ (lower coefficient in grade II, Amide II), $1530 \mathrm{~cm}^{-1}$ (lower coefficient in grade II, $\mathrm{C}=\mathrm{N}$ and/or $\mathrm{C}=\mathrm{C}$ stretching), $1512 \mathrm{~cm}^{-1}$ (higher coefficient in grade II, C-H in-plane bending), $1481 \mathrm{~cm}^{-1}$ (lower coefficient in grade II, Amide II), $1454 \mathrm{~cm}^{-1}$ (higher coefficient in grade II, asymmetric methyl deformation), $1396 \mathrm{~cm}^{-1}$ (higher coefficient in grade II, symmetric $\mathrm{CH}_{3}$ bending of the methyl groups of proteins) and $1068 \mathrm{~cm}^{-1}$ (higher coefficient in grade II, $\mathrm{C}-\mathrm{O}$ stretching in ribose) [12]. Nine spectral markers with absolute coefficients $>0.01$ were found in the DBM spectrum for grade II (+ coefficients) versus grade I recurrence (- coefficients) (Fig. 2e): $1708 \mathrm{~cm}^{-1}$ (lower coefficient in grade II, $\mathrm{C}=\mathrm{O}$ stretching in thymine), $1643 \mathrm{~cm}^{-1}$ (lower coefficient in grade II, Amide I), $1624 \mathrm{~cm}^{-1}$ (higher coefficient in grade II, base carbonyl stretching and ring breathing mode in nucleic acids), $1600 \mathrm{~cm}^{-1}$ (lower coefficient in grade II, $\mathrm{C}=\mathrm{O}$ stretching in lipids), $1512 \mathrm{~cm}^{-1}$ (higher coefficient in grade II, C-H in-plane bending), $1490 \mathrm{~cm}^{-1}$ (lower coefficient in grade II, $\mathrm{C}=\mathrm{C}$ and/or in-plane $\mathrm{C}-\mathrm{H}$ bending), $1454 \mathrm{~cm}^{-1}$ (higher coefficient in grade II, asymmetric methyl deformation), $1339 \mathrm{~cm}^{-1}$ (higher coefficient in grade II, collagen) and $1068 \mathrm{~cm}^{-1}$ (higher coefficient in grade II, C-O stretching in ribose) [12]. The DBM spectrum for grade I recurrence (+ coefficients) versus grade I (- coefficients) meningiomas (Fig. 1f) indicates 10 spectral markers with absolute coefficients > 0.01: $1698 \mathrm{~cm}^{-1}$ (higher coefficient in grade I recurrence, $\mathrm{C}_{2}=\mathrm{O}$ stretching in guanine), $1663 \mathrm{~cm}^{-1}$ (lower coefficient in grade $\mathrm{I}$ recurrence, $\mathrm{C}=\mathrm{O}$ stretching in cytosine), $1647 \mathrm{~cm}^{-1}$ (higher coefficient in grade I recurrence, Amide I), $1624 \mathrm{~cm}^{-1}$ (lower coefficient in grade I recurrence, base carbonyl stretching and ring breathing mode in nucleic acids), $1550 \mathrm{~cm}^{-1}$ (lower coefficient in grade I recurrence, Amide II), $1527 \mathrm{~cm}^{-1}$ (lower coefficient in grade I recurrence, $\mathrm{C}=\mathrm{N}$ and/ or $\mathrm{C}=\mathrm{C}$ stretching), $1496 \mathrm{~cm}^{-1}$ (higher coefficient in grade I recurrence, $\mathrm{C}=\mathrm{C}$ stretching and $/$ or $\mathrm{C}-\mathrm{H}$ bending), $1460 \mathrm{~cm}^{-1}$ (lower coefficient in grade I recurrence, asymmetric $\mathrm{CH}_{3}$ bending in collagen), $1393 \mathrm{~cm}^{-1}$ (higher coefficient in grade I recurrence, symmetric $\mathrm{CH}_{3}$ bending in proteins) and $1335 \mathrm{~cm}^{-1}$ (lower coefficient in grade I recurrence, $\mathrm{CH}$ ring deformation in polysaccharides or pectin) [12].

The spectral data underwent chemometric analysis by means of PCA-LDA, as a first discriminant attempt, and then by PLS-DA as a final and best discriminant model. The following comparisons were investigated: (1) grade I versus grade II meningioma, (2) grade I versus grade I meningiomas that re-occurred and (3) grade II versus grade I meningiomas that re-occurred.

\section{Grade I versus grade II meningiomas}

The pre-processed data were initially separated into two subsets: training ( $70 \%$ of the samples) and validation (30\% of the samples) using the KS algorithm. The training set was used for model construction while the validation set for final model
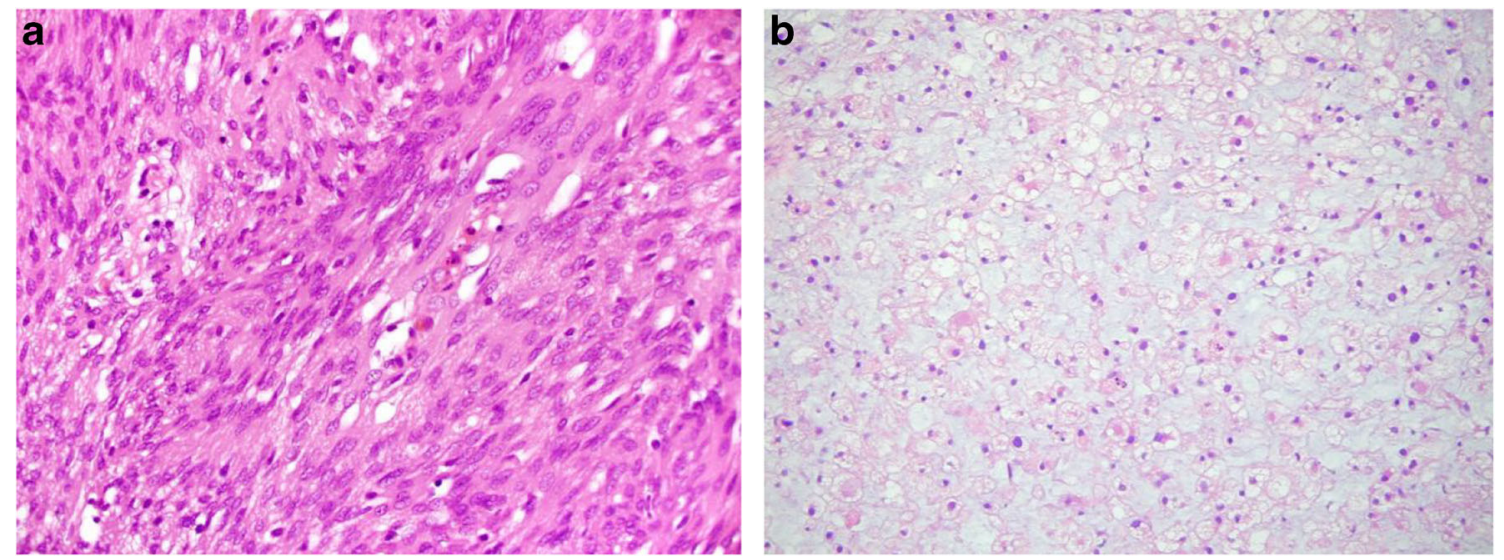

Fig. 1 H\&E slides. (a) WHO grade I meningioma (transitional meningioma). (b) WHO grade II meningioma (clear cell) 
Fig. 2 Infrared spectra for meningioma tumour samples (grade I, grade I recurrence and grade II). (a) Raw spectra and (b) pre-processed spectra (SavitzkyGolay $2^{\text {nd }}$ derivative and vector normalisation), where black line represents mean spectrum. (c) Mean spectrum for each class overlaid. (d) Difference-betweenmean (DBM) spectrum for grade II (+) vs. grade I (-) meningiomas. (e) DBM spectrum for grade II (+) vs. grade I recurrence (-) meningiomas. (f) DBM spectrum for grade I recurrence $(+)$ vs. grade I (-) meningiomas, where solid dots represent spectral wavenumbers with absolute coefficients $>0.01$

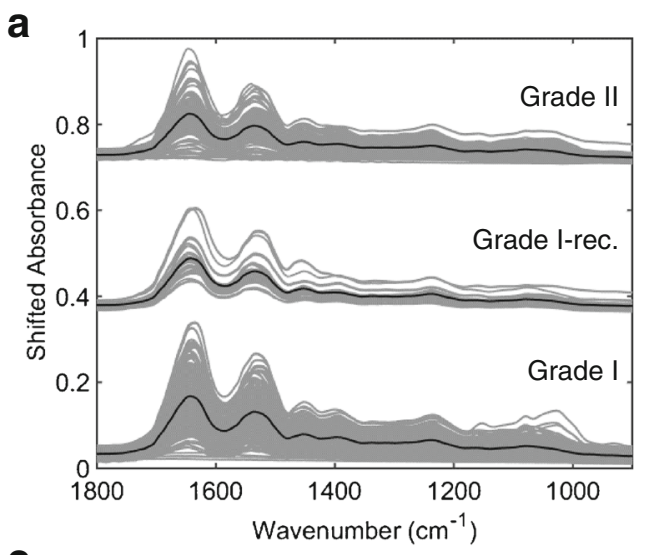

C

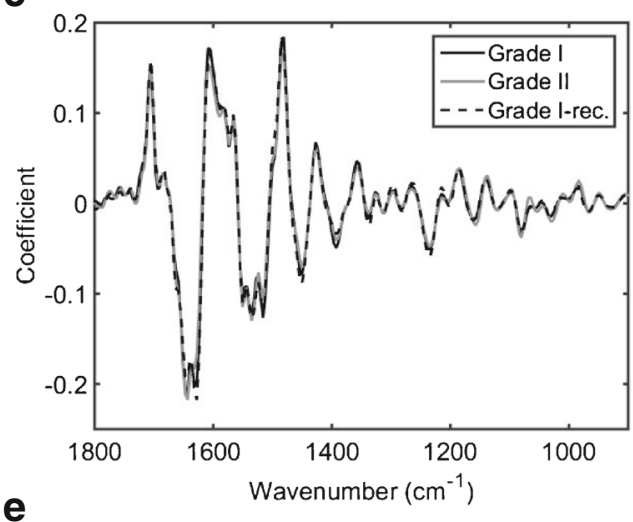

e

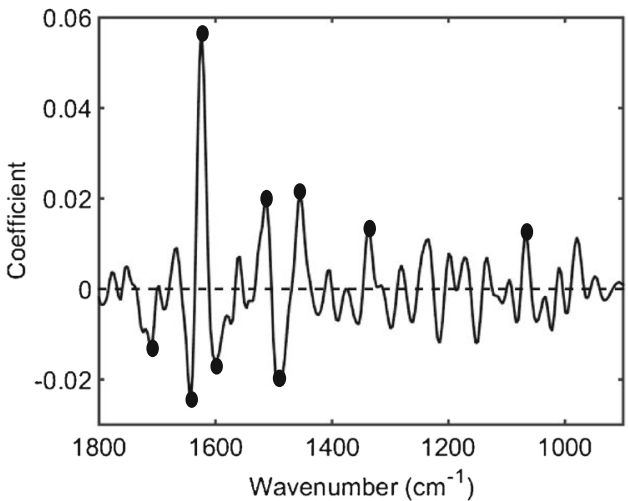

b

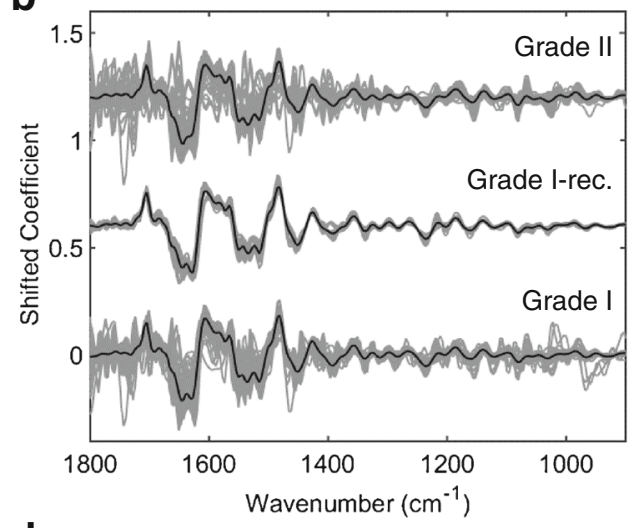

d
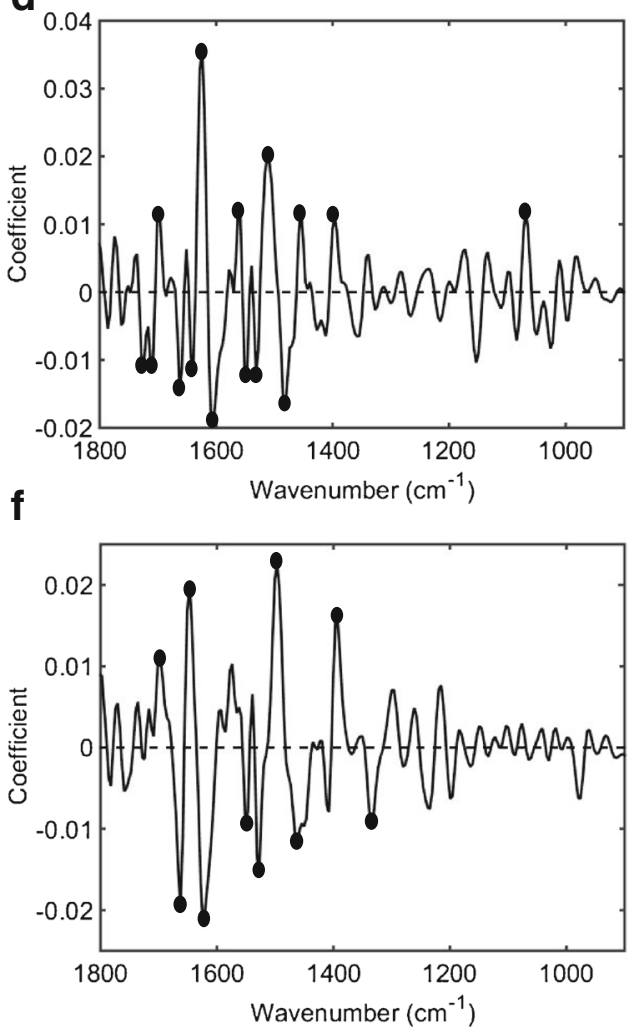

evaluation. PCA-LDA was applied to the spectral data using 10 PCs (98\% explained variance, see ESM Fig. S5), where training and validation accuracies were estimated at $89 \%$ and $71 \%$, respectively (Table 1). Despite having reasonable accuracies and sensitivity ( $89 \%$ in the validation set), the

Table 1 Quality metrics for PCA-LDA and PLS-DA models to distinguish grade I vs. grade II samples

\begin{tabular}{lllll}
\hline Algorithm & Dataset & Accuracy (\%) & Sensitivity (\%) & Specificity (\%) \\
\hline PCA-LDA & Training & 89 & 98 & 62 \\
& Validation & 71 & 89 & 20 \\
\multirow{2}{*}{ PLS-DA } & Training & 97 & 96 & 99 \\
& Validation & 79 & 80 & 73 \\
\hline
\end{tabular}

specificity in validation was $20 \%$, indicating that many grade I meningiomas were predicted as grade II. PLS-DA was then applied to the spectral data as a most powerful alternative for class differentiation. PLS-DA model was built with $11 \mathrm{LVs}$ (93\% spectral explained variance, see ESM Fig. S5), generating accuracies of $97 \%$ and $79 \%$ in the training and validation sets, respectively (Table 1 ). The sensitivity and specificity in the validation set were equal to $80 \%$ and $73 \%$, respectively, with an area under the curve (AUC) value equal to 0.82 , which indicates a good classification model. The PLS-DA discriminant function (DF) graph and receiver operating characteristic (ROC) curve to discriminate grade I and grade II meningiomas are depicted in Fig. 3. PLS-DA coefficients (see ESM Fig. S6) were used to extract biomarker information through an automatic peak detection algorithm that sought for the 8 

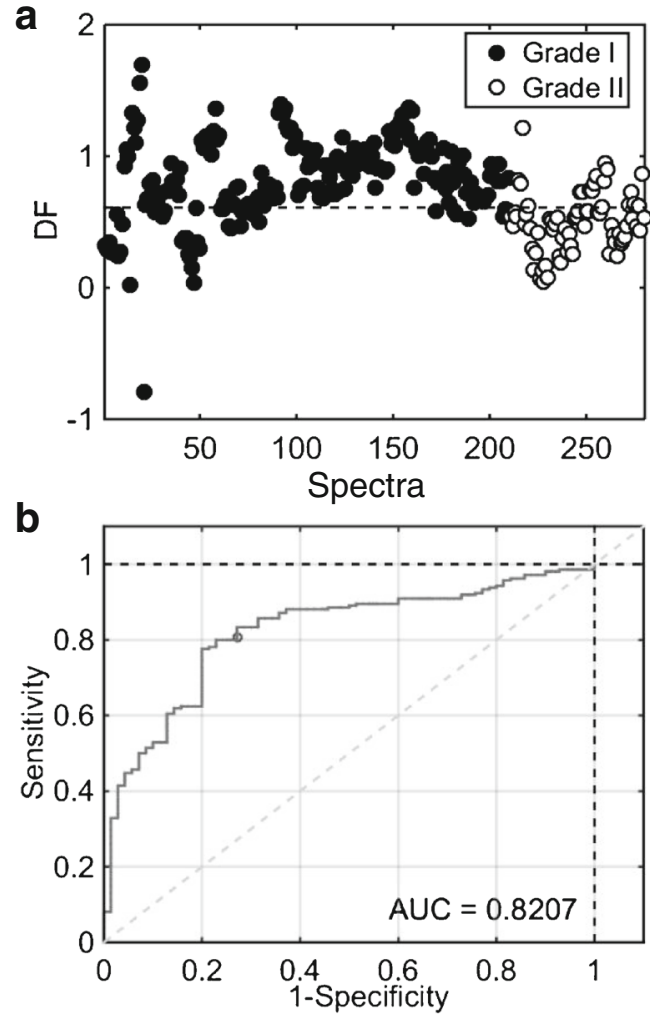

Fig. 3 PLS-DA results to distinguish grade I vs. grade II meningiomas. (a) Discriminant function (DF) plot for samples' spectra. (b) Receiver operating characteristic (ROC) curve, where AUC stands for area under the curve

most relevant peaks representing the wavenumbers with highest absolute coefficients (Table 2). PCA-LDA and PLSDA model residuals are shown in ESM Fig. S4a and b.

\section{Grade I versus grade I meningiomas that re-occurred}

Cross-validated PCA-LDA was applied to the spectral data using 17 PCs (97\% explained variance) (ESM Fig. S7), where
Table 3 Quality metrics for PCA-LDA and PLS-DA models to distinguish grade I vs. grade I recurrence samples

\begin{tabular}{lllll}
\hline Algorithm & Dataset & Accuracy (\%) & Sensitivity (\%) & Specificity (\%) \\
\hline PCA-LDA & Training & 95 & 99 & 34 \\
& Validation & 95 & 99 & 32 \\
\multirow{2}{*}{ PLS-DA } & Training & 96 & 96 & 100 \\
& Validation & 94 & 94 & 94 \\
\hline
\end{tabular}

both training and validation accuracies were estimated at $95 \%$ (Table 3). Despite having excellent values of accuracy and sensitivity (99\%), the specificity is again low in validation (32\%), indicating that many grade I meningiomas were predicted as grade I that re-occurred. PLS-DA was applied to the spectral data with 17 LVs (95\% spectral explained variance) (see ESM Fig. S7), generating accuracies of $96 \%$ and $94 \%$ in the training and validation sets, respectively (Table 3 ). The sensitivity and specificity in the validation were both equal to $94 \%$, with an AUC value equal to 0.98 , which indicates an almost perfect classification. The PLS-DA DF graph and ROC curve to discriminate grade I and grade I recurrence meningiomas are depicted in Fig. 4. The wavenumbers with highest absolute PLS-DA coefficients (see ESM Fig. S8) are depicted in Table 4. PCA-LDA and PLS-DA model residuals are shown in Fig. S4c and S4d (see ESM).

\section{Grade II versus grade I meningiomas that re-occurred}

Cross-validated PCA-LDA was applied to the spectral data using 12 PCs (96\% explained variance) (see ESM Fig. S9), where both training and validation accuracies were estimated at $90 \%$ (Table 5). Once more, the specificity of PCA-LDA is highly affected (45\%), despite having the good accuracies and sensitivity (99\%). PLS-DA was applied to the spectral data with 13 LVs (95\% spectral explained variance) (see ESM
Table 2 Spectral markers identified by PLS-DA in order to discriminate grade I and grade II meningiomas

\begin{tabular}{llll}
\hline Wavenumber $\left(\mathrm{cm}^{-1}\right)$ & Tentative assignment & Relative intensity $^{\mathrm{a}}$ & $P$ value \\
\hline 1651 & Amide I & $\downarrow$ & $0.035\left(^{*}\right)$ \\
1593 & $\mathrm{NH}_{2}$ adenine & $\downarrow$ & $<10^{-7}(* *)$ \\
1546 & Amide II & $\uparrow$ & 0.637 \\
1500 & $\delta(\mathrm{CH})$ in-plane & $\uparrow$ & $<10^{-4}(* *)$ \\
1454 & $\delta\left(\mathrm{CH}_{3}\right)$ asymmetric & $\downarrow$ & $<10^{-13}(* *)$ \\
1377 & $v(\mathrm{C}-\mathrm{O})$ & $\downarrow$ & $0.030(*)$ \\
1227 & $v_{\text {as }}\left(\mathrm{PO}_{2}{ }^{-}\right)$ & $\downarrow$ & 0.051 \\
1122 & $v(\mathrm{C}-\mathrm{O})$ in carbohydrates & $\uparrow$ & $0.014(*)$ \\
\hline
\end{tabular}

$P$ value was calculated by an ANOVA test

$\delta$ bending, $v$ stretching, $v_{\text {as }}$ asymmetric stretching

$* P$ value $<0.05$ considered statistically significant; $* * P$ value $<0.001$ considered statistically highly significant

$\mathrm{a} \uparrow=$ higher intensity in grade II meningioma; $\downarrow=$ lower intensity in grade II meningioma 

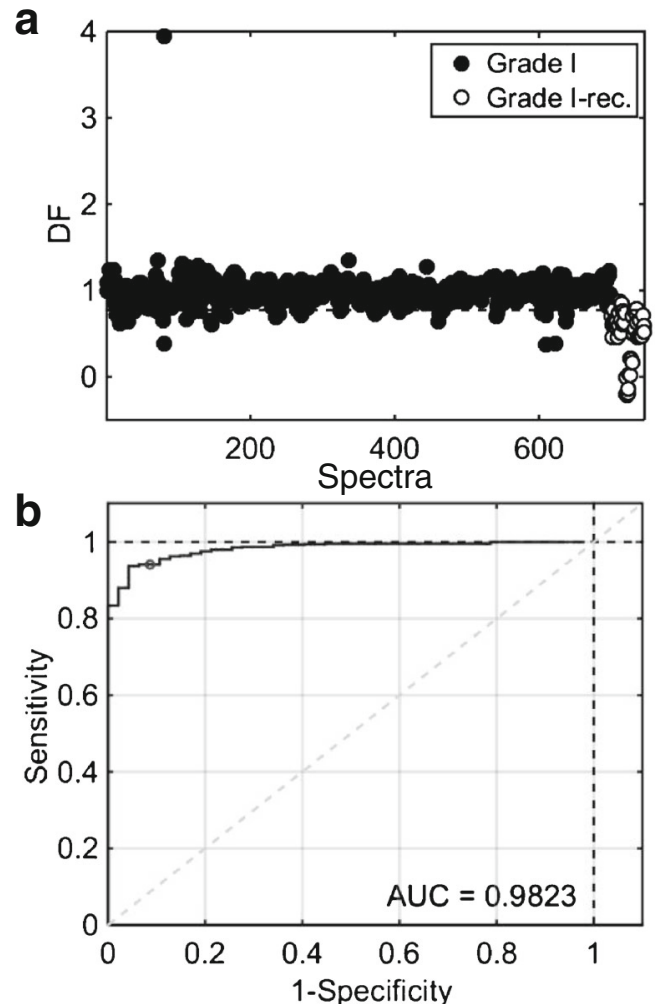

Fig. 4 PLS-DA results to distinguish grade I vs. grade I recurrence meningiomas. (a) Discriminant function (DF) plot for samples' spectra. (b) Receiver operating characteristic (ROC) curve, where AUC stands for area under the curve

Fig. S9), generating accuracies of $99 \%$ and $97 \%$ in the training and validation sets, respectively (Table 5). The sensitivity and specificity in the validation were both equal to $97 \%$, with an AUC value equal to 0.99 , which indicates a close-to-perfect classification. The PLS-DA DF graph and ROC curve to discriminate grade II and grade I recurrence meningiomas are depicted in Fig. 5, where the wavenumbers with highest absolute PLS-DA coefficients are depicted in Table 6. PCA-
Table 5 Quality metrics for PCA-LDA and PLS-DA models to distinguish grade II vs. grade I recurrence samples

\begin{tabular}{lllll}
\hline Algorithm & Dataset & Accuracy (\%) & Sensitivity (\%) & Specificity (\%) \\
\hline PCA-LDA & Training & 90 & 99 & 47 \\
& Validation & 90 & 98 & 45 \\
\multirow{2}{*}{ PLS-DA } & Training & 99 & 98 & 100 \\
& Validation & 97 & 97 & 100 \\
\hline
\end{tabular}

LDA and PLS-DA model residuals are shown in Fig. S4e and S4f (see ESM).

\section{Discussion}

Normal and tumour brain tissues have been previously discriminated using IR or Raman spectroscopy [1], where neoplastic tissues (meningioma, glioma and brain metastasis) were found to be statically significant from normal tissues using PCA-LDA as the multivariate spectral analysis technique. Hands et al. [30] reported serum diagnostic of brain tumours using ATR-FTIR spectroscopy with support vector machines (SVMs) with the sensitivity of $89.4 \%$ and specificity of $78.0 \%$ to distinguish cancerous from non-cancerous samples and the sensitivity of $82.1 \%$ and specificity of $75.0 \%$ to distinguish glioma from meningioma tissue. Bury et al. [31] reported the use of ATR-FTIR spectroscopy to analyse plasma samples in order to distinguish non-cancer from different cancerous brain samples. Normal and meningioma samples were differentiated with $87 \%$ accuracy using PCA-LDA and 95\% accuracy using SVM, and meningioma samples were diagnosed among several groups of samples (normal, high-grade glioma, low-grade glioma and brain metastasis) with an accuracy of $63 \%$ using PCA-LDA and an accuracy of $100 \%$ using SVM.
Table 4 Spectral markers identified by PLS-DA in order to discriminate grade I and grade I recurrence meningiomas

\begin{tabular}{llll}
\hline Wavenumber $\left(\mathrm{cm}^{-1}\right)$ & Tentative assignment & Relative intensity $^{\mathrm{a}}$ & $P$ value \\
\hline 1755 & $v(\mathrm{C}=\mathrm{O})$ in lipids & $\downarrow$ & $<10^{-3}(* *)$ \\
1693 & Amide I (antiparallel $\beta$-sheet $)$ & $\uparrow$ & $<10^{-3}(* *)$ \\
1477 & $\delta\left(\mathrm{CH}_{2}\right)$ in lipids & $\downarrow$ & 0.229 \\
1423 & $\delta\left(\mathrm{CH}_{2}\right)$ in polysaccharides & $\uparrow$ & 0.722 \\
1400 & $v_{\mathrm{s}}\left(\mathrm{COO}^{-}\right)$in amino acids (aspartate, glutamate) & $\downarrow$ & $<10^{-5}(* *)$ \\
1369 & $\left(\mathrm{C}^{-\mathrm{N}}\right)$ in cytosine and guanine & $\downarrow$ & 0.542 \\
1346 & $\delta\left(\mathrm{CH}_{2}\right)$ in collagen & $\downarrow$ & 0.940 \\
1246 & $v_{\text {as }}\left(\mathrm{PO}_{2}{ }^{-}\right)$ & $\uparrow$ & $<10^{-4}(* *)$ \\
\hline
\end{tabular}

$P$ value was calculated by an ANOVA test

$\delta$ bending, $v$ stretching, $v_{\mathrm{s}}$ symmetric stretching, $v_{\text {as }}$ asymmetric stretching

$* * P$ value $<0.001$ considered statistically highly significant

$\mathrm{a} \uparrow=$ higher intensity in grade I recurrence meningioma; $\downarrow=$ lower intensity in grade I recurrence meningioma 

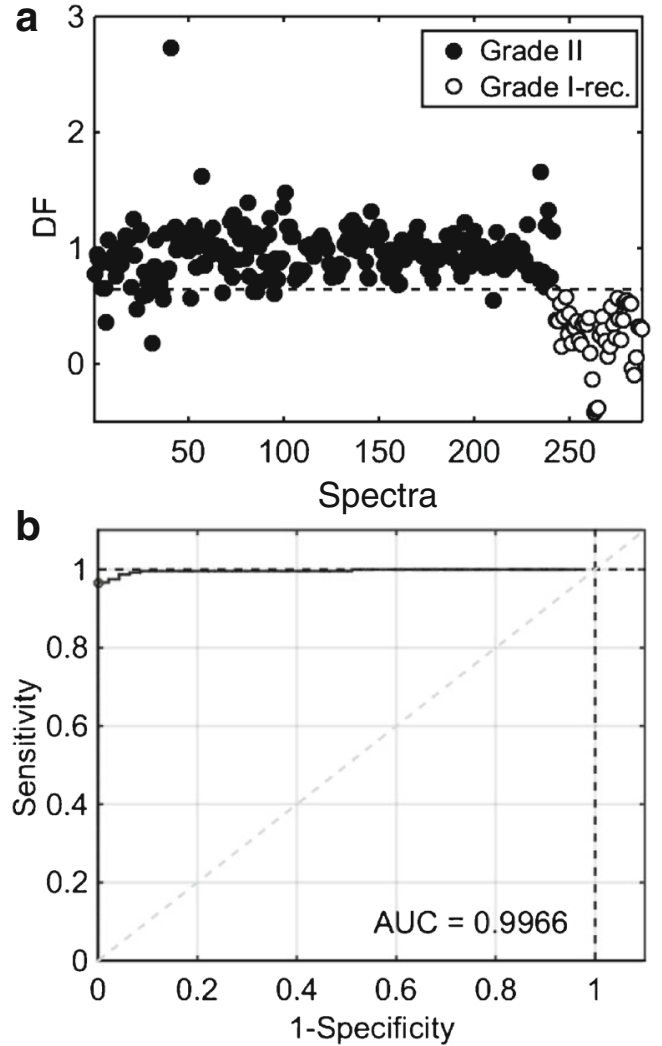

Fig. 5 PLS-DA results to distinguish grade II vs. grade I recurrence meningiomas. (a) Discriminant function (DF) plot for samples' spectra. (b) Receiver operating characteristic (ROC) curve, where AUC stands for area under the curve

Herein, WHO grade I and grade II meningiomas were discriminated with $79 \%$ accuracy in the validation set $(80 \%$ sensitivity, $73 \%$ specificity, AUC $=0.82$ ) using PLS-DA, a simpler and less susceptible method to overfitting than SVM, indicating a satisfactory clinical performance taking into consideration the complexity of the data obtained, as demonstrated by the patient demographics in Table S1 (see ESM) and the inherent spectrochemical complexity of tissue samples. Despite having high accuracies and sensitivities, the lower specificities of the
PCA-LDA models indicate that these models are skewed towards the bigger class size, so the model is classifying the samples from this class more accurately (high sensitivity) than the samples from the smaller class (low specificity). The accuracy is influenced by the class size, so it tends to follow the sensitivity. The PLS-DA models, on the other hand, have a better consistency between sensitivity and specificity, thus indicating no overfitting. The statistically significant spectral markers were mainly associated with proteins (Amide I, Amide II), carbohydrates $(v(\mathrm{C}-\mathrm{O}))$ and DNA/RNA functional groups $\left(\mathrm{NH}_{2}\right.$ adenine, $\left.v_{\text {as }}\left(\mathrm{PO}_{2}{ }^{-}\right)\right)$(Table 2). Proteins play an important role in the molecular pathways for meningiomas, where, for example, integrin exhibits different expression profiles within different grades of meningioma [32]. In addition, Amide I, Amide II and carbohydrate absorptions have been associated with differences between normal and meningioma tissues [31], and $\delta(\mathrm{CH}), \delta\left(\mathrm{CH}_{3}\right), v(\mathrm{C}-\mathrm{O})$ and $v_{\text {as }}\left(\mathrm{PO}_{2}{ }^{-}\right)$have been found to be related to spectral markers associated with brain tumours in general [30]. These findings indicate that IR spectroscopy allied with chemometrics could be used to aid clinical differentiation of grade I and grade II meningioma tumours in a non-destructive, fast and sensitive way.

"Grade I" and "grade I recurrence" were found to be clearly different, in which a discriminant performance of $94 \%$ accuracy (94\% sensitivity and specificity) was obtained to distinguish both types of tumours. This indicates that one can assess the presence of recurrence in comparison with regular grade I tumours in an objective and automatic fashion by using IR spectroscopy and chemometrics. This is immensely important stratification information, which cannot be routinely derived or inferred from the histological examination of meningiomas lying within a WHO grade. The spectral markers associated with recurrence (Table 4) were mainly protein (Amide I), lipids, collagen and DNA/RNA changes $\left(v_{\mathrm{as}}\left(\mathrm{PO}_{2}^{-}\right)\right)$. DNA alterations, in special DNA methylation, are highly associated with meningioma progression, especially as a discriminant feature between NF2-mutated and non-NF2-mutated tumours [33]. By evaluating the spectral profile of all patients in grade
Table 6 Spectral markers identified by PLS-DA in order to discriminate grade II and grade I recurrence meningiomas

\begin{tabular}{llll}
\hline Wavenumber $\left(\mathrm{cm}^{-1}\right)$ & Tentative assignment & Relative intensity & $P$ value \\
\hline 1639 & Amide I & $\downarrow$ & 0.579 \\
1597 & $\mathrm{NH}_{2}$ adenine & $\uparrow$ & $0.001\left(^{*}\right)$ \\
1547 & Amide II & $\downarrow$ & 0.425 \\
1523 & $v(\mathrm{C}=\mathrm{N})$ & $\uparrow$ & $0.018(*)$ \\
1454 & $\delta\left(\mathrm{CH}_{3}\right)$ asymmetric & $\uparrow$ & $<10^{-3}(* *)$ \\
1265 & $v_{\text {as }}\left(\mathrm{PO}_{2}^{-}\right)$ & $\uparrow$ & $<10^{-11}(* *)$ \\
1242 & $\mathrm{Ammide} \mathrm{III}^{-}$ & $\uparrow$ & $<10^{-5}(* *)$ \\
1122 & $v(\mathrm{C}-\mathrm{O})$ in carbohydrates & $\downarrow$ & 0.251 \\
\hline$P$ value was calculated by an ANOVA test & & \\
$* P$ value $<0.05$ considered statistically significant; $* * P$ value $<0.001$ considered statistically highly significant \\
a $\uparrow=$ higher intensity in grade I recurrence meningioma; $\downarrow=$ lower intensity in grade I recurrence meningioma
\end{tabular}


I cohort, 12 patients (patients 2, 11, 16, 22, 26, 30, 34, 38, 50, 56,57 and 69) were found to have these spectral markers following the same trend observed in grade I recurrence, in terms of relative intensity. This corresponds to $17 \%$ of grade I cohort, and these patients could be potential candidates to have grade I re-occurring in the future, once their spectral marker profiles are similar to the ones in grade I recurrence cohort. In this case, these patients could be followed closely in the clinical scenario to evaluate if the tumour will re-occur in the future. Since meningioma re-occurs with an average time of 10 years, this pilot study does not have this confirmative information for these patients, although the estimated recurrence rate of $17 \%$ is close to the usual meningioma grade I recurrence rate of $10 \%$. This is just a hypothesis that needs further validation, but if this methodology is proved true, one could use this spectrochemical information to follow up patients with higher likelihood of recurrence and provide them with more specific treatments and closer attention, reducing existent costs associated with unknown recurrence odds.

Finally, grade II and grade I recurrence were discriminated based on their spectrochemical profile with an accuracy of $97 \%$ (97\% sensitivity and $100 \%$ specificity) and the main spectral markers associated with recurrence (Table 6) were proteins (Amide I, Amide II and Amide III), carbohydrates ( $v(\mathrm{C}-\mathrm{O})$ ) and DNA/RNA alterations $\left(\mathrm{NH}_{2}\right.$ adenine, $\left.v_{\text {as }}\left(\mathrm{PO}_{2}{ }^{-}\right)\right)$, therefore indicating that these tumour types are very different. An important advantage of using ATR-FTIR spectroscopy is that due to its non-destructive nature [34], the same tissue section could theoretically be used for conventional histological analysis or other complementary techniques such as Raman microspectroscopy [35]. Moreover, the sensitivity and specificity for meningioma tumour detection towards clinical diagnosis might improve in future applications using FTIR microspectroscopy due to its relatively larger spatial resolution in comparison with ATR-FTIR spectroscopy, which enables the acquisition of richer spatially distributed spectrochemical information.

Acknowledgements C.L.M. Morais would like to thank the Coordenação de Aperfeiçoamento de Pessoal de Nível Superior (CAPES), Brazil (grant 88881.128982/2016-01), for his research grant. The authors would like to thank the Brain Tumour North West (BTNW) for providing the samples. H\&E images are also a courtesy from the BTNW biobank.

\section{Compliance with ethical standards}

Conflict of interest The authors declare that they have no conflict of interest.

Open Access This article is licensed under a Creative Commons Attribution 4.0 International License, which permits use, sharing, adaptation, distribution and reproduction in any medium or format, as long as you give appropriate credit to the original author(s) and the source, provide a link to the Creative Commons licence, and indicate if changes were made. The images or other third party material in this article are included in the article's Creative Commons licence, unless indicated otherwise in a credit line to the material. If material is not included in the article's Creative Commons licence and your intended use is not permitted by statutory regulation or exceeds the permitted use, you will need to obtain permission directly from the copyright holder. To view a copy of this licence, visit http://creativecommons.org/licenses/by/4.0/.

\section{References}

1. Gajjar K, Heppenstall LD, Pang W, Ashton KM, Trevisan J, Patel II, et al. Diagnostic segregation of human brain tumours using Fourier-transform infrared and/or Raman spectroscopy coupled with discriminant analysis. Anal Methods. 2013;5:89-102.

2. Davis ME. Epidemiology and overview of gliomas. Semin Oncol Nurs. 2018;34:420-9.

3. Mehta K, Atak A, Sahu A, Srivastava S, Murali KC. An early investigative serum Raman spectroscopy study of meningioma. Analyst. 2018;143:1916-23.

4. Takahashi Y, Wanibuchi M, Kimura Y, Akiyama Y, Mikami T, Mikuni N. Meningioma originating from the hypoglossal canal: case report and review of literature. World Neurosurg. 2019;127: 525-9.

5. Yeo Y, Park C, Lee JW, Kang Y, Ahn JM, Kang HS, et al. Magnetic resonance imaging spectrum of spinal meningioma. Clin Imaging. 2019;55:100-6.

6. Miller R Jr, DeCandio ML, Dixon-Mah Y, Giglio P, Vandergrift WA III, Banik NL, et al. Molecular targets and treatment of meningioma. J Neurol Neurosurg. 2014;1:1000101.

7. Wrobel G, Roerig P, Kokocinski F, Neban K, Hahn M, Reifenberger G, et al. Microarray-based gene expression profiling of benign, atypical and anaplastic meningiomas identifies novel genes associated with meningioma progression. Int J Cancer. 2005;114:249-56.

8. Saraf S, McCarthy BJ, Villano JL. Update on meningiomas. Oncologist. 2011;16:1604-13.

9. Wernicke AG, Dicker AP, Whiton M, Ivanidze J, Hyslop T, Hammond EH, et al. Assessment of epidermal growth factor receptor (EGFR) expression in human meningioma. Radiat Oncol. 2010;5:46.

10. Baker MJ, Trevisan J, Bassan P, Bhargava R, Butler HR, Dorling $\mathrm{KM}$, et al. Using Fourier transform IR spectroscopy to analyze biological materials. Nat Protoc. 2014;9:1771-91.

11. Martin FL, Kelly JG, Llabjani V, Martin-Hirsch PL, Patel II, Trevisan J, et al. Distinguishing cell types or populations based on the computational analysis of their infrared spectra. Nat Protoc. 2010;5:1748-60.

12. Movasaghi Z, Rehman S, ur Rehman I. Fourier transform infrared (FTIR) spectroscopy of biological tissues. Appl Spectrosc Rev. 2008;43:134-79.

13. Kelly JG, Trevisan J, Scott AD, Carmichael PL, Pollock HM, Martin-Hirsch PL, et al. Biospectroscopy to metabolically profile biomolecular structure: a multistage approach linking computational analysis with biomarkers. J Proteome Res. 2011;10:1437-48.

14. Morais CLM, Paraskevaidi M, Cui L, Fullwood NJ, Isabelle M, Lima KMG, et al. Standardization of complex biologically derived spectrochemical datasets. Nat Protoc. 2019;14:1546-77.

15. Morais CLM, Lima KMG. Principal component analysis with linear and quadratic discriminant analysis for identification of cancer samples based on mass spectrometry. J Braz Chem Soc. 2018;29: 472-81.

16. Brereton RG, Lloyd GR. Partial least squares discriminant analysis: taking the magic away. J Chemom. 2014;28:213-25. 
17. Kenig S, Bedolla DE, Birarda G, Faoro V, Mitri E, Vindigni A, et al. Fourier transform infrared microspectroscopy reveals biochemical changes associated with glioma stem cell differentiation. Biophys Chem. 2015;207:90-6.

18. Wehbe K, Forfar I, Eimer S, Cinque G. Discrimination between two different grades of human glioma based on blood vessel infrared spectral imaging. Anal Bioanal Chem. 2015;407:7295-305.

19. Beleites C, Steiner G, Sowa MG, Baumgartner R, Sobottka S, Schackert G, et al. Classification of human gliomas by infrared imaging spectroscopy and chemometric image processing. Vib Spectrosc. 2005;38:143-9.

20. Amharref N, Beljebbar A, Dukic S, Venteo L, Schneider L, Plout $\mathrm{M}$, et al. Brain tissue characterisation by infrared imaging in a rat glioma model. Biochim Biophys Acta-Biomembranes. 1758;2006: 892-9.

21. Wehbe K, Pineau R, Eimer S, Vital A, Loiseau H, Déléris G. Differentiation between normal and tumor vasculature of animal and human glioma by FTIR imaging. Analyst. 2010;135:3052-9.

22. Noreen R, Pineau R, Chin CC, Cestelli-Guidi M, Hwu Y, Marcelli A, et al. Functional histology of glioma vasculature by FTIR imaging. Anal Bioanal Chem. 2011;401:795-801.

23. Steiner G, Shaw A, Choo-Smith LP, Abuid MH, Schackert G, Sobottka S, et al. Distinguishing and grading human gliomas by IR spectroscopy. Biopolymers. 2003;72:464-71.

24. Ali K, Lu Y, Christensen C, May T, Hyett C, Griebel R, et al. Fourier transform infrared spectromicroscopy and hierarchical cluster analysis of human meningiomas. Int J Mol Med. 2008;21:297301.

25. Paraskevaidi M, Morais CLM, Raglan O, Lima KMG, Paraskevaidis E, Martin-Hirsch PL, et al. Aluminium foil as an alternative substrate for the spectroscopic interrogation of endometrial cancer. J Biophotonics. 2018;11:e201700372.

26. Ballabio D, Consonni V. Classification tools in chemistry. Part 1: linear models. PLS-DA. Anal Methods. 2013;5:3790-8.
27. Kennard RW, Stone LA. Computer aided design of experiments. Technometrics. 1969;11:137-48.

28. Bro R, Smilde AK. Principal component analysis. Anal Methods. 2014;6:2812-31.

29. Morais CLM, Lima KMG. Comparing unfolded and twodimensional discriminant analysis and support vector machines for classification of EEM data. Chemometr Intell Lab Syst. 2017;170:1-12.

30. Hands JR, Clemens G, Stables R, Ashton K, Brodbelt A, Davis C, et al. Brain tumour differentiation: rapid stratified serum diagnostics via attenuated total reflection Fourier-transform infrared spectroscopy. J Neuro-Oncol. 2016;127:463-72.

31. Bury D, Morais CLM, Paraskevaidi M, Ashton KM, Dawson TP, Martin FL. Spectral classification for diagnosis involving numerous pathologies in a complex clinical setting: a neuro-oncology example. Spectrochim Acta A Mol Biomol Spectrosc. 2019;206:89-96.

32. Abbritti RV, Polito F, Cucinotta M, Lo Giudice C, Caffo M, Tomasello C, et al. Meningiomas and proteomics: focus on new potential biomarkers and molecular pathways. Cancer Genomics Proteomics. 2016;13:369-79.

33. Suppiah S, Nassiri F, Bi WL, Dunn IF, Hanemann CO, Horbinski $\mathrm{CM}$, et al. Molecular and translational advances in meningiomas. Neuro Oncol. 2019;21(Supplement_1):i4-i17.

34. Maitra I, Morais CLM, Lima KMG, Ashton KM, Date RS, Martin FL. Attenuated total reflection Fourier-transform infrared spectral discrimination in human bodily fluids of oesophageal transformation to adenocarcinoma. Analyst. 2019;144:7447-56.

35. Morais CLM, Lilo T, Ashton KM, Davis C, Dawson TP, Gurusinghe N, et al. Determination of meningioma brain tumour grades using Raman microspectroscopy imaging. Analyst. 2019;144:7024-31.

Publisher's note Springer Nature remains neutral with regard to jurisdictional claims in published maps and institutional affiliations. 